\title{
RESEARCH
}

\section{Local treatment for focal progression in metastatic neuroendocrine tumors} \author{
Daniel A Anaya ${ }^{1}$ and Jonathan Strosberg ${ }^{1}$ \\ 'Department of GI Oncology, H. Lee Moffitt Cancer Center and Research Institute, Tampa, Florida, USA \\ 2Pancreas Translational \& Clinical Research Center, Ospedale San Raffaele IRCCS, Milano, Italy \\ 3Università, Vita e Salute, Milan, Italy \\ ${ }^{4}$ Department of Biomedical Sciences and Human Oncology, University of Bari, Bari, Italy
}

Taymeyah Al-Toubah1, Stefano Partelli2,3, Mauro Cives4, Valentina Andreasi², Franco Silvestris4, Massimo Falconi2,3

Correspondence should be addressed to J Strosberg: jonathan.strosberg@moffitt.org

\begin{abstract}
New systemic treatments have improved the therapeutic landscape for patients with metastatic gastroenteropancreatic neuroendocrine tumors (GEP-NETs). While drugs such as everolimus, sunitinib, temozolomide and 177Lutetium-dotatate are appropriate for patients with widespread disease progression, local treatment approaches may be more appropriate for patients with unifocal progression. Surgical resection, radiofrequency ablation (RFA), hepatic arterial embolization (HAE) or radiation, can control discrete sites of progression, allowing patients to continue their existing therapy and sparing them toxicities of a new systemic treatment. We identified 69 patients with metastatic GEP-NETs who underwent a local treatment for focal progression in the setting of widespread metastases. Twenty-six percent underwent resection, 27\% RFA, 23\% external beam radiation and $23 \%$ selective HAE. With a median follow-up of 25 months, 42 (61\%) patients subsequently progressed to the point of requiring additional intervention (12 locoregional, 30 systemic) for disease control. Median time to new systemic treatment was 32 months ( $95 \% \mathrm{Cl}, 16.5-47.5$ months). Median time to any additional intervention was 19 months (95\% Cl, 8.7-25.3 months). Control of local sites of progression enabled the majority of patients to remain on their existing systemic treatment and avoid potential toxicities associated with salvage systemic therapy.
\end{abstract}

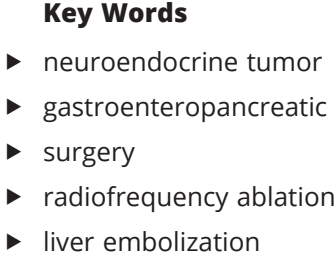

Endocrine-Related Cancer (2019) 26, 405-409

\section{Introduction}

Gastroenteropancreatic neuroendocrine tumors (GEP-NETs) are a heterogeneous group of tumors which include NETs of the gastrointestinal tract and pancreas. Tumor grade and differentiation are important prognostic factors. Low-grade tumors are defined as having a mitotic rate of $0-1$ per ten high powered fields (HPF) or Ki-67 index of $0-2 \%$, intermediate-grade tumors having a mitotic rate of 2-20 or Ki-67 index of 3-20\% and highgrade tumors having a mitotic rate or Ki-67 index $>20 \%$ (Lloyd et al. 2017). Although well-differentiated GEP-NETs are often slow growing, even indolent metastatic tumors can become more aggressive and change grade over the disease course, likely due to accumulation of mutations (Rinke et al. 2009, Yang et al. 2011, Tang et al. 2016, Wong et al. 2018).

The therapeutic landscape for GEP-NETs has significantly improved over the past decade with several systemic therapeutic options for patients with widely metastatic disease. These include somatostatin analogs (SSAs), mTOR and angiogenesis inhibitors, radiolabeled 
SSAs and cytotoxic chemotherapy (Raymond et al. 2011, Strosberg et al. 2011, Caplin et al. 2014, Yao et al. 2016a,b, Panzuto et al. 2017, Pavel et al. 2017, Strosberg et al. 2017, Kunz et al. 2018). While SSAs have minimal associated toxicities, most systemic therapies are associated with significant risks and side effects.

Locoregional treatment options, such as surgery, ablation and radiation, can be useful for managing oligometastases. These treatment options are typically reserved for patients with limited metastases or for hepatic debulking. However, they can be appropriately used in certain instances to target individual aggressive tumors in patients with otherwise stable metastatic disease.

While the literature offers data on treatment options in the setting of widespread progression as well as upfront treatment for newly diagnosed patients, there is no published data on approaches to managing progression of individual metastases in the setting of widespread metastatic disease. Our study presents retrospective data on patients with this particular situation and measures the duration of disease control by evaluating progressionfree survival (PFS) and time to new systemic therapy.

\section{Patients and methods}

We reviewed the records of patients seen between January 2014 and February 2018 with metastatic GEP-NETs at three NET referral centers: Moffitt Cancer Center in Florida, USA, Pancreatic Surgery, San Raffaele Hospital, Milan, Italy and the University of Bari in Bari, Italy. Study protocols (containing waiver of consent due to the retrospective nature of the studies) were approved by the appropriate ethics committee at each institution. Chart reviews and existing institutional databases were used to identify all patients with metastatic GEP-NET who underwent a nonsystemic treatment for progression of solitary tumor(s) (no more than two) at any metastatic site in the setting of widespread metastatic disease. Progression could have been documented using any diagnostic modality, including cross-sectional or somatostatin receptor imaging. Patients undergoing regional (lobar) or whole liver directed embolization, as well as liver debulking surgery were not included in this analysis.

\section{Data collection}

Demographic and pathological data were collected from each patient, including date and stage of initial diagnosis, histologic grade, Ki-67 proliferation and mitotic count, primary disease site, location(s) of metastatic disease, location of treated tumor, oncologic therapy history, symptoms associated with locoregional therapy, most recent follow-up date, vital status and date of death. Dates of all interventions and therapies were also collected, along with dates of progression on said therapies.

\section{Statistical analysis}

PFS was defined as time from initial locoregional therapy to radiographic progression (at any site). Progression status was assessed based on review of medical notes and scan reports, not based on formal Response Evaluation Criteria in Solid Tumors (RECIST) radiographic readings. Overall survival (OS) was measured from date of initial diagnosis until death from any cause or last known follow-up. Time to systemic treatment was defined as time from initial locoregional therapy to initiation of new systemic treatment. Time to any intervention was defined as time from initial locoregional therapy to time of any new anti-cancer intervention (locoregional, systemic, surgical, etc.). Survival curves were estimated using the Kaplan-Meier method and compared by the log-rank test. Exact 95\% confidence intervals (CIs) were calculated for each proportion of interest. Statistical significance was declared when $P<0.05$. All statistical analyses were performed using SPSS, version 24.

\section{Results}

\section{Patient and tumor characteristics}

Table 1 presents the demographics and tumor characteristics of the 69 patients on the study. Overall, the majority of patients had either pancreatic $(n=38$, $55 \%)$ or small intestinal $(n=26,38 \%)$ primary tumors. The remaining $7 \%(n=5)$ of patients had primary tumors in the cecum, rectum and stomach; two had unknown primaries. All but four tumors were well differentiated, and most $(n=43,62 \%)$ were low grade.

\section{Prior treatments}

All subjects had received at least one prior treatment at the time they were selected to undergo the locoregional treatment. Patients received a median of three prior treatments (range 1-12) including two prior systemic therapies (range 0-9). Only four patients had not received prior systemic therapy at time of selection. 
Table 1 Patient demographics and tumor characteristics.

\begin{tabular}{llc}
\hline Gender, $n(\%)$ & Male & $41(59.4)$ \\
& Female & $28(40.6)$ \\
Location of primary, $n(\%)$ & Pancreas & $38(55)$ \\
& Small intestine & $26(37.6)$ \\
& Unknown & $2(2.9)$ \\
& Cecum & $1(1.4)$ \\
& Rectum & $1(1.4)$ \\
& Stomach & $1(1.4)$ \\
Grade, $n$ (\%) & Low & $43(62.3)$ \\
& Intermediate & $19(27.5)$ \\
& High & $4(5.8)$ \\
Location of treated tumor, & Unknown & $3(4.3)$ \\
$n(\%)$ & Liver & $52(75.4)$ \\
& Lymph node & $6(8.7)$ \\
& Ovary & $6(8.7)$ \\
& Lung & $2(2.9)$ \\
& Peritoneum & $2(2.9)$ \\
& Bone & $1(1.4)$ \\
Locoregional therapy, $n$ (\%) & Ablation & $19(27.5)$ \\
& Surgical & $18(26.1)$ \\
& resection & \\
& Embolization & $16(23.2)$ \\
& Radiation & $16(23.2)$ \\
\hline
\end{tabular}

Three of the patients (one pancreatic, two small bowel) were asymptomatic with low-volume liver metastases who developed progression of a solitary liver tumor and the fourth patient was a cecal NET patient with lowvolume liver metastases (status-post debulking) who developed solitary right ovarian progression. The prior therapies varied from somatostatin analogs $(n=61,92 \%)$, chemotherapy $(n=34,49 \%)$, radiation therapy $(n=4,6 \%)$, surgical resection(s) $(n=46,67 \%)$, embolizations $(n=16$, $23 \%)$, radiolabeled SSA $(n=10,14 \%)$, everolimus $(n=14$, $20 \%)$, sunitinib $(n=5,7 \%)$ and clinical trial drugs $(n=3$, $4 \%)$. Eight percent $(n=55)$ of patients continued on their systemic therapy after completing their locoregional treatment. In most of these cases $(n=45,65 \%)$, the systemic therapy consisted of SSA monotherapy.

\section{Locoregional treatments}

After multidisciplinary discussion, subjects underwent one of four locoregional interventions: surgical resection

Table 2 Adverse events post locoregional therapy.

\begin{tabular}{ll}
\hline Adverse event & $\boldsymbol{n}(\%)$ \\
\hline Pain & $6(8.7)$ \\
Diarrhea & $3(4.3)$ \\
Vomiting & $2(2.9)$ \\
Skin irritation & $1(1.4)$ \\
Cholecystitis & $1(1.4)$ \\
Infection & $1(1.4)$
\end{tabular}

( $n=18,26 \%)$, embolization $(n=16,23 \%)$, ablation $(n=19$, $27 \%)$ or external beam radiation $(n=16,23 \%)$. The target treated local tumors were primarily solitary liver metastases $(n=52,75 \%)$. Other sites of treated metastases included lymph nodes $(n=6)$, ovarian metastases $(n=6)$, peritoneal nodules $(n=2)$, lung nodules $(n=2)$ and bone metastases $(n=1)$.

Of the patients that received external beam radiation, sites of treated metastases included lymph nodes $(n=6)$, liver metastases $(n=6)$, peritoneal nodules $(n=2)$, lung nodules $(n=1)$ and bone metastases $(n=1)$. Six of the patients (three liver, two peritoneal, one lymph node) progressed (at a median of 14 months) following locoregional treatment and received multiple systemic therapies. Three patients (one liver, two lymph nodes) had durable responses that lasted a median of 27 months before they ultimately progressed and received systemic treatment (2) or subsequent surgical resection (1). The remaining patients have not progressed further to the point of requiring subsequent therapeutic intervention.

Only 20\% $(n=14)$ of subjects experienced adverse events associated with the locoregional therapy, presented in Table 2. All adverse events were acute in nature and resolved very shortly after presentation with minimal or no intervention. Twenty percent of patients reported substantial improvement in symptoms following treatment.

\section{Subsequent interventions and therapies}

Of the 69 treated subjects, 42 (61\%) patients subsequently progressed to the point of requiring additional intervention (12 locoregional, 30 systemic) for disease control. 9/18 patients with surgically resected metastases eventually progressed requiring subsequent intervention, 8 of whom required subsequent systemic therapy. $12 / 16$ of those who had liver embolizations required further intervention, 8 of whom required subsequent systemic therapy. 11/19 of those who had ablations required further intervention, 6 of whom required subsequent systemic therapy. 10/16 of those who had radiation required further intervention, 8 of whom required subsequent systemic therapy.

Subsequent systemic therapies included cytotoxic chemotherapy $(n=7,10 \%)$, everolimus $(n=6,9 \%)$, sunitinib $(n=7,10 \%)$, radiolabeled SSA $(n=3,4 \%)$, clinical trial drugs $(n=3,4 \%)$, standard dose SSA $(n=3,4 \%)$ and doseescalated SSA $(n=1,1 \%)$. Among patients who underwent a second locoregional treatment after progression $(n=14)$, six patients received liver embolization (9\%), five patients underwent surgical resection upon progression (7\%), two patients had RFAs and one underwent radiation. 
Of the 18 patients who underwent surgical resection, eight exhibited increase in tumor grade and seven had an absolute increase in Ki-67 > than 5\% as compared with the primary diagnosis. None of the resection specimens were described as poorly differentiated even if Ki-67 was above $20 \%$.

\section{PFS and OS}

With a median follow-up of 25 months, (range, 1-123 months), 11 patients died. Median OS was not reached in this analysis. The survival rate was $97 \%$ at 2 years.

Median PFS was 17 months (95\% CI, 8.7-25.3 months; Fig. 1). Median time to any additional intervention was 19 months (95\% CI, 11.9-26.1 months) and median time to new systemic treatment was 32 months (95\% CI, 16.5-47.5 months).

Median time to new systemic therapy for patients undergoing RFAs ( $n=6,32 \%)$ was 39 months compared to 17 months for patients undergoing embolizations $(n=8$, $50 \%)$. Those undergoing surgical resection $(n=8,44 \%)$ and radiation therapy $(n=8,50 \%)$ had median time to systemic therapy of 21 and 32 months, respectively. None of these differences were statistically significant.

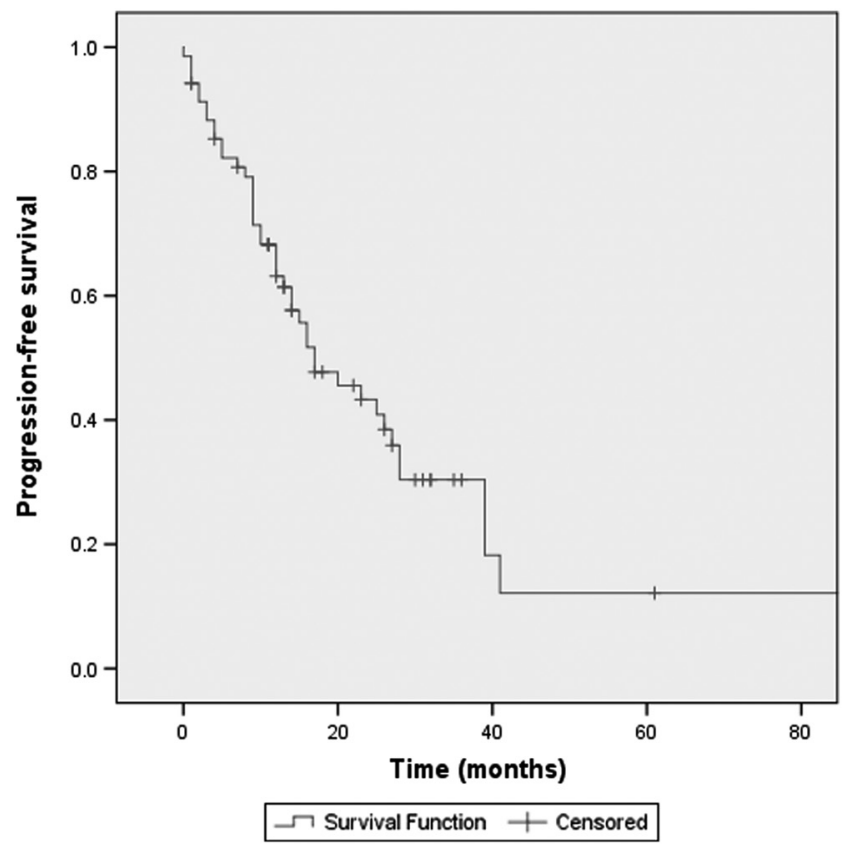

Figure 1

Progression-free survival Kaplan-Meier curve.

\section{Discussion}

Growth of individual tumors in the setting of widely metastatic disease is not an unusual circumstance in metastatic NETs, as illustrated in this study. In this situation, locoregional rather than systemic treatment is often selected in order to target the enlarging tumor and delay introduction of a new, and potentially toxic, systemic treatment.

While there are substantial data published on systemic management of progressive NETs, there have been no studies evaluating the management of unifocal progression in a widespread metastatic setting. Our study included patients who underwent selective radiation, surgical resection, RFA or HAE to discrete sites of progression. We did not include patients who underwent lobar HAEs or cytoreductive hepatic surgery.

All patients with the exception of two had metastatic disease to the liver and 62 of 67 patients had metastatic disease at initial diagnosis. There was no significant difference between type of locoregional therapy and time to initiation of new systemic therapy. None of the patients experienced any long-term side effects requiring further intervention or treatment.

The locoregional treatments evaluated in this study were able to delay the need for further systemic therapy by a median of 32 months and yielded a median PFS of 17 months. The time to any additional intervention was a median of 19 months. These relatively long durations of disease control validate the treatment strategy of targeting progressive tumors and delaying change in systemic treatment. Our data also support the concept that treatment decisions should be made after review of each individual case in a multidisciplinary tumor board setting and ideally at a specialized NET center.

Limitations of this study include its retrospective methodology, heterogeneity of enrolled patients and lack of RECIST analysis of progression. However, it is important to note that RECIST methodology, with its sampling of lesions in various organs, is not designed to address the particular circumstance of unifocal disease progression.

\section{Conclusions}

We identified a large cohort of patients with metastatic GEP-NETs who underwent a local treatment for unifocal progression in the setting of widespread metastases. These tumors were both clinically and pathologically aggressive, as confirmed by significant elevations in Ki-67 index 
in surgically resected tumors. Control of local sites of progression enabled the majority of patients to remain on their existing systemic treatment (or on no treatment) and avoid potential toxicities associated with salvage systemic therapy.

\section{Declaration of interest}

J Strosberg: Novartis (Consult), Ipsen and Lexicon (Speaker bureau). The other authors have nothing to disclose.

\section{Funding}

This research did not receive any specific grant from any funding agency in the public, commercial or not-for-profit sector.

\section{References}

Caplin ME, Pavel M, Cwikla JB, Phan AT, Raderer M, Sedlackova E, Cadiot G, Wolin EM, Capdevila J, Wall L, et al. 2014 Lanreotide in metastatic enteropancreatic neuroendocrine tumors. New England Journal of Medicine 371 224-233. (https://doi.org/10.1056/ NEJMoa1316158)

Kunz PL, Catalano PJ, Nimeiri H, Fisher GA, Longacre TA, Suarez CJ, Yao JC, Kulke MH, Hendifar AE, Shanks JC, et al. 2018 A randomized study of temozolomide or temozolomide and capecitabine in patients with advanced pancreatic neuroendocrine tumors: a trial of the ECOGACRIN Cancer Research Group (E2211). Journal of Clinical Oncology 36 4004-4004. (https://doi.org/10.1200/JCO.2018.36.15_suppl.4004)

Lloyd RV, Osamura RY, Kloppel G \& Rosai J 2017 WHO Classification of Tumours of Endocrine Organs. Lyon, France: IARC Press.

Panzuto F, Cicchese N, Partelli S, Rinzivillo M, Capurso G, Merola E, Manzoni M, Pucci E, Iannicelli E, Pilozzi E, et al. 2017 Impact of Ki67 re-assessment at time of disease progression in patients with pancreatic neuroendocrine neoplasms. PLOS ONE 12 e0179445. (https://doi.org/10.1371/journal.pone.0179445)

Pavel ME, Baudin E, Oberg KE, Hainsworth JD, Voi M, Rouyrre N, Peeters M, Gross DJ \& Yao JC 2017 Efficacy of everolimus plus octreotide LAR in patients with advanced neuroendocrine tumor and carcinoid syndrome: final overall survival from the randomized, placebo-controlled phase 3 RADIANT-2 study. Annals of Oncology 28 1569-1575. (https://doi.org/10.1093/annonc/mdx193)

Raymond E, Dahan L, Raoul JL, Bang YJ, Borbath I, Lombard-Bohas C, Valle J, Metrakos P, Smith D, Vinik A, et al. 2011 Sunitinib malate for the treatment of pancreatic neuroendocrine tumors. New England Journal of Medicine 364 501-513. (https://doi.org/10.1056/ NEJMoa1003825)

Rinke A, Muller HH, Schade-Brittinger C, Klose KJ, Barth P, Wied M, Mayer C, Aminossadati B, Pape UF, Blaker M, et al. 2009 Placebocontrolled, double-blind, prospective, randomized study on the effect of octreotide LAR in the control of tumor growth in patients with metastatic neuroendocrine midgut tumors: a report from the PROMID Study Group. Journal of Clinical Oncology 27 4656-4663. (https://doi.org/10.1200/JCO.2009.22.8510)

Strosberg JR, Fine RL, Choi J, Nasir A, Coppola D, Chen DT, Helm J \& Kvols L 2011 First-line chemotherapy with capecitabine and temozolomide in patients with metastatic pancreatic endocrine carcinomas. Cancer 117 268-275. (https://doi.org/10.1002/ cncr.25425)

Strosberg J, El-Haddad G, Wolin E, Hendifar A, Yao J, Chasen B, Mittra E, Kunz PL, Kulke MH, Jacene H, et al. 2017 Phase 3 trial of 177Lu-Dotatate for midgut neuroendocrine tumors. New England Journal of Medicine 376 125-135. (https://doi.org/10.1056/ NEJMoa1607427)

Tang LH, Untch BR, Reidy DL, O'Reilly E, Dhall D, Jih L, Basturk O, Allen PJ \& Klimstra DS 2016 Well-differentiated neuroendocrine tumors with a morphologically apparent high-grade component: a pathway distinct from poorly differentiated neuroendocrine carcinomas. Clinical Cancer Research 22 1011-1017. (https://doi. org/10.1158/1078-0432.CCR-15-0548)

Wong HL, Yang KC, Shen Y, Zhao EY, Loree JM, Kennecke HF, Kalloger SE, Karasinska JM, Lim HJ, Mungall AJ, et al. 2018 Molecular characterization of metastatic pancreatic neuroendocrine tumors (PNETs) using whole-genome and transcriptome sequencing. Cold Spring Harbor Molecular Case Studies 4 a002329. (https://doi. org/10.1101/mcs.a002329)

Yang Z, Tang LH \& Klimstra DS 2011 Effect of tumor heterogeneity on the assessment of Ki67 labeling index in well-differentiated neuroendocrine tumors metastatic to the liver: implications for prognostic stratification. American Journal of Surgical Pathology 35 853-860. (https://doi.org/10.1097/PAS.0b013e31821a0696)

Yao JC, Fazio N, Singh S, Buzzoni R, Carnaghi C, Wolin E, Tomasek J, Raderer M, Lahner H, Voi M, et al. $2016 a$ Everolimus for the treatment of advanced, non-functional neuroendocrine tumours of the lung or gastrointestinal tract (RADIANT-4): a randomised, placebo-controlled, phase 3 study. Lancet 387 968-977. (https://doi org/10.1016/S0140-6736(15)00817-X)

Yao JC, Pavel M, Lombard-Bohas C, Van Cutsem E, Voi M, Brandt U, He W, Chen D, Capdevila J, de Vries EGE, et al. 2016b Everolimus for the treatment of advanced pancreatic neuroendocrine tumors: overall survival and circulating biomarkers from the randomized, phase III RADIANT-3 study. Journal of Clinical Oncology 34 3906-3913. (https://doi.org/10.1200/JCO.2016.68.0702)

Received in final form 2 January 2019

Accepted 18 January 2019

Accepted Preprint published online 22 January 2019 (c) 2019 Society for Endocrinology Published by Bioscientifica Ltd. Printed in Great Britain 